\title{
HYPERFINITE-DIMENSIONAL SUBSPACES OF THE NONSTANDARD HULL OF $c_{0}$
}

\author{
L. C. MOORE, JR.
}

\begin{abstract}
Let $\hat{c}_{0}$ be the nonstandard hull of the Banach space $c_{0}$ formed with respect to an $\boldsymbol{x}_{1}$-saturated extension. Then $\hat{c}_{0}$ is not isometrically isomorphic to any hyperfinite-dimensional subspace of $\hat{c}_{0}$ and hence not to any hyperfinite-dimensional Banach space. This gives a negative answer to the question posed by Ward Henson: "Does every Banach space have a nonstandard hull which is isometrically isomorphic to a hyperfinite-dimensional Banach space?" As a consequence of the result, no ultrapower of $c_{0}$ is isometrically isomorphic to an ultraproduct of finite-dimensional Banach spaces.
\end{abstract}

1. Introduction. Let $E$ be a real Banach space considered as an element of a set-theoretical structure $\mathfrak{R}$ and let $\hat{E}$ be the nonstandard hull of $E$ with respect to an $\aleph_{1}$-saturated extension $* \mathfrak{T}$. In particular $\hat{E}$ may be an ultrapower of $E$. Ward Henson showed that the following statements are equivalent [4].

(a) $E$ has a nonstandard hull which is isometrically isomorphic to a hyperfinitedimensional Banach space.

(b) $E$ has an ultrapower which is isometrically isomorphic to an ultraproduct of finite-dimensional Banach spaces.

(c) For every positive bounded sentence $\sigma$ which is true in $E$ and each positive integer $m$, there is a finite-dimensional Banach space in which $\sigma_{m}^{+}$is true.

In addition he showed in [3] and [4] that each of the Banach spaces $l_{p}$, $1<p<\infty$, has a nonstandard hull which is isometrically isomorphic to a hyperfinite-dimensional Banach space and asked whether this was true for every Banach space. The main result in this paper is that the sequence space $c_{0}$ does not have a nonstandard hull isometrically isomorphic to a hyperfinite-dimensional Banach space. (The argument given here is for the space of real sequences, but a slight variation extends the result to the complex case.) The author wishes to thank Ward Henson and the referee for many helpful suggestions.

2. Preliminaries. Recall that the nonstandard hull of a Banach space $E$ is the vector space quotient $\hat{E}$ of $\operatorname{fin}\left({ }^{*} E\right)=\left\{p \in{ }^{*} E:{ }^{*}\|p\|\right.$ is finite $\}$ by $\mu(0)=\{p \in$ ${ }^{*} E:{ }^{*}\|p\|$ is infinitesimal $\}$. If $\pi$ is the canonical quotient map of $\operatorname{fin}\left({ }^{*} E\right)$ onto $\hat{E}$, then the norm on $\hat{E}$ is given by $\|\pi(p)\|=\operatorname{st}\left({ }^{*}\|p\|\right)$ where st is the standard part map. Since $* \Re$ is $\aleph_{1}$-saturated, $\hat{E}$ is a Banach space in which $E$ may be embedded by the identification of $\pi\left({ }^{*} x\right)$ with $x$ for all $x$ in $E$.

Received by the editors November 6, 1979; presented to the Society, January 6, 1980. AMS (MOS) subject classifications (1970). Primary 46B99, 02H25; Secondary 26A98. Key words and phrases. Nonstandard hulls, ultraproducts, ultrapowers. 
Let $\mathscr{F}$ be the family of all finite-dimensional Banach spaces in $\mathscr{N}$. If $W$ is an element of $* \mathscr{F}$, then the vector space quotient $\hat{W}$ of $\operatorname{fin}(W)=\left\{p \in W:{ }^{*}\|p\|\right.$ is finite $\}$ by $\mu_{W}(0)=\left\{p \in W:{ }^{*}\|p\|\right.$ is infinitesimal $\}$ is again a Banach space under the norm $\|\pi(p)\|=\operatorname{st}\left({ }^{*}\|p\|\right)$, where $\pi$ now denotes the quotient map of $\operatorname{fin}(W)$ onto $\hat{W}$. Such a Banach space is called a hyperfinite-dimensional Banach space. For basic information on nonstandard hulls and details of the nonstandard extension the reader is referred to [5]. For a good summary of work in ultrapowers and ultraproducts the reader is referred to [2].

Let $L$ be a first order language whose nonlogical symbols are a binary function symbol + , two unary predicate symbols $P$ and $Q$ and for each rational number $r$ a unary function symbol $f_{r}$. Each real Banach space is regarded as an $L$-structure by taking $+_{E}$ to be addition on $E$, by setting $P_{E}=\{x:\|x\|<1\}$ and $Q_{E}=\{x$ : $\|x\|>1\}$ and by taking $\left(f_{r}\right)_{E}$ to be the operation of scalar multiplication by $r$ for each rational number $r$. A term $t$ in $L$ may be considered as an expression of the form $r_{1} x_{1}+r_{2} x_{2}+\cdots+r_{n} x_{n}$ where $r_{1}, r_{2}, \ldots, r_{n}$ are rational and $x_{1}, x_{2}, \ldots, x_{n}$ are variables. Then the interpretations in a Banach space $E$ of the atomic formulas $P(t)$ and $Q(t)$ with $a_{1}, a_{2}, \ldots, a_{n}$ assigned to $x_{1}, x_{2}, \ldots, x_{n}$ are $\| r_{1} a_{1}+r_{2} a_{2}$ $+\cdots+r_{n} a_{n} \|<1$ and $\left\|r_{1} a_{1}+r_{2} a_{2}+\cdots+r_{n} a_{n}\right\|>1$ respectively. A positive bounded formula in $L$ is one which can be built up from atomic formulas using conjunction, disjunction and the bounded quantifiers $(\exists x)(P x \wedge \ldots)$ and $(\forall x)$ $(P x \rightarrow \ldots)$. Now let $\sigma$ be a positive bounded formula of $L$ and let $m$ be a positive integer. We obtain $\sigma_{m}^{+}$from $\sigma$ as follows: for atomic formulas (i) replace $t=s$ by $P(m \cdot(t-s))$; (ii) replace $P(t)$ by $P((1-(1 / m)) \cdot t)$; (iii) replace $Q(t)$ by $Q((1+(1 / m)) \cdot t)$. For more complex formulas let $(\sigma \wedge \tau)_{m}=\sigma_{m}^{+} \wedge \tau_{m}^{+},(\sigma \vee \tau)_{m}^{+}$ $=\sigma_{m}^{+} \vee \tau_{m}^{+},(\exists x)(P x \wedge \sigma)_{m}^{+}=(\exists x)\left(P x \wedge \sigma_{m}^{+}\right),(\forall x)(P x \rightarrow \sigma)_{m}^{+}=(\forall x)$ $\left(P x \rightarrow \sigma_{m}^{+}\right)$and proceed inductively. For details the reader is referred to [4].

3. Examples. The property of $\hat{c}_{0}$ which is fundamental in proving our main result is that for each $x$ of norm 1 there is a $y$ of norm 1 such that both $x+y$ and $x-y$ have norm 1. Certainly no finite-dimensional Banach space satisfies this. Moreover this condition is easily formulated as a positive bounded sentence $\sigma$. Thus using the equivalence of (a) and (c) above, one approach to the main result would be to find an integer $n$ such that $\sigma_{n}^{+}$is false in every finite-dimensional space. However this cannot be done; for each $n$ there is a finite-dimensional space $F_{n}$ such that for each $x$ of norm 1 in $F_{n}$ there exists a $y$ of norm 1 in $F_{n}$ such that both $\|x+y\|$ and $\|x-y\|$ lie between $1-1 / n$ and $1+1 / n$.

To construct such spaces let $n$ be fixed and let $\left\{f_{j}: 1<j<n+1\right\}$ be sequences of the form

$$
f_{j}=(\underbrace{0, \ldots, 0,1,0, \ldots, 0}_{n+1 \text { places }}, \underbrace{ \pm 1 / n, \pm 1 / n, \ldots, \pm 1 / n}_{2^{n+1} \text { places }}, 0,0, \ldots)
$$

such that

(i) for each $j, f_{j}(j)=1$ and $f_{j}(k)=0$ for $k \neq j, k=1,2, \ldots, n+1$.

(ii) for each finite sequence $\varepsilon_{1}, \varepsilon_{2}, \ldots, \varepsilon_{n+1}$ of $+1,-1$ there is a $k$ such that $f_{j}(k)=\varepsilon_{j} \cdot 1 / n$ for all $j$. 
Let $F_{n}=\operatorname{span}\left\{f_{1}, f_{2}, \ldots, f_{n+1}\right\}$ in $c_{0}$. So if $f$ is in $F_{n}$ with $\|f\|=1$, then $f=\sum_{k=1}^{n+1} c_{j} f_{j}$ where $\left|c_{j}\right|<1$ for all $j$. Also $\left|c_{j}\right|>1 / n$ for at most $n$ of the $j$ 's, since there exists $k$ such that $f(k)=(1 / n) \sum_{j=1}^{n+1}\left|c_{j}\right|$. Pick $j_{0}$ so that $\left|c_{j_{0}}\right|<1 / n$. For $f+f_{j_{0}}$, $1-1 / n<1-\left|c_{j_{0}}\right|<\left|\left(f+f_{j_{0}}\right)\left(j_{0}\right)\right|<1+\left|c_{j_{0}}\right|<1+1 / n$. If $k \neq j_{0}$, then $\left|\left(f+f_{j_{0}}\right)(k)\right|<|f(k)|+1 / n<1+1 / n$. Hence $1-1 / n<\left\|f+f_{j_{0}}\right\|<1+1 / n$ and similarly $1-1 / n<\left\|f-f_{j_{0}}\right\|<1+1 / n$.

Note that these examples give rise to a hyperfinite-dimensional Banach space $\hat{W} \subseteq \hat{c}_{0}$ which satisfies the condition $\sigma$ above. In particular the unit ball of $\hat{W}$ has no extreme points. Namely take $W$ to be ${ }^{*} F_{\omega}$ where $\omega$ is an infinite integer. Hence to prove the main result we must use more information about $\hat{c}_{0}$. In the argument below the lattice structure of $\hat{c}_{0}$ plays a crucial role.

\section{Proof of theorem.}

THEOREM. The nonstandard hull $\hat{c}_{0}$ of $c_{0}$ is not isometrically isomorphic to any hyperfinite-dimensional Banach space.

Since $c_{0}$ is a Banach lattice, $\hat{c}_{0}$ is also a Banach lattice under the natural extension of the order [1]. Indeed $\hat{c}_{0}$ may be considered as a closed sublattice of $l_{\infty}\left({ }^{*} N\right)$. To see this recall that ${ }^{*} c_{0}$ consists of all internal functions $p$ from ${ }^{*} N$ to ${ }^{*} R$ such that for every $0<r$ in ${ }^{*} R$ there exists $n$ in ${ }^{*} N$ such that $|p(k)|<r$ for all $k>n$. In particular for some $k_{0}$ in ${ }^{*} N,\|p\|=\left|p\left(k_{0}\right)\right|=\max \left\{|p(k)|: k \in{ }^{*} N\right\}$. The element $p$ is in fin $\left({ }^{*} c_{0}\right)$ if and only if this maximum is finite and $p$ is in $\mu(0)$ if and only if this maximum is infinitesimal. Thus if $p$ and $q$ are in $\operatorname{fin}\left({ }^{*} c_{0}\right)$, then $\pi(p)=\pi(q)$ exactly when $\operatorname{st}(p(k))=\operatorname{st}(q(k))$ for all $k$ in $* N$. It follows that each element $\pi(p)$ of $\hat{c}_{0}$ can be considered as an element of $l_{\infty}\left({ }^{*} N\right)$ by setting $\pi(p)(k)=$ $\operatorname{st}(p(k))$ for $k$ in ${ }^{*} N$. Clearly the norm on $\pi(p)$ is the same as the norm in $l_{\infty}\left({ }^{*} N\right)$. Indeed under this identification, $\hat{c}_{0}$ is just the closed span of the characteristic functions of *-finite sets. In the following the letters $p$, $q$, etc. represent elements of ${ }^{*} c_{0}$ so that $p(k)$ and $q(k)$ are elements of ${ }^{*} R$, while $x, y$, etc. represent elements of $\hat{c}_{0}$ so that $x(k)$ and $y(k)$ are standard real numbers.

Recall that a nonzero element $a$ of a Banach lattice $L$ is called an atom if for each $u$ in $L$ with $0<u<|a|$ there exists a real number $\theta$ such that $u=\theta a$. It is easy to see that the atoms of $\hat{c}_{0}$ are simply nonzero multiples of the characteristic functions of singleton sets in ${ }^{*} N$.

LEMMA 1. An element $e$ of $\hat{c}_{0}$ is an atom of norm 1 if and only if $\|e\|=1$ and there do not exist $x, y$ in $\hat{c}_{0}$ such that $\|x\|=1,0<\|y\|<1, x+y=e,\|x+(1 /\|y\|) y\|$ $=\|x-(1 /\|y\|) y\|=1$.

Proof of Lemma 1. Let $e$ be an atom of $\hat{c}_{0}$ of norm 1. We may assume that $e$ is the characteristic function of $\{k\}$ for some $k$ in ${ }^{*} N$. Suppose there exist $x, y \in \hat{c}_{0}$ with $\|x\|=1,0<\|y\| \leqslant 1, x+y=e$ and $\|x+(1 /\|y\|) y\|=\|x-(1 /\|y\|) y\|$ $=1$. If $x(k)=-1$, then $y(k)=2$ and $\|y\|$ is not less than or equal to 1 . If $|x(k)|<1$, then there exists $k^{\prime} \neq k$ such that $\left|x\left(k^{\prime}\right)\right|=1$. So $y\left(k^{\prime}\right)=-x\left(k^{\prime}\right)$ and $\|x-y\|=2$ which is a contradiction. Thus $x(k)=1$. Now pick $k_{0}$ such that 
$\left|y\left(k_{0}\right)\right|=\|y\|$. Note $k_{0} \neq k$, so $x\left(k_{0}\right)=-y\left(k_{0}\right)$ and $\left|x\left(k_{0}\right)-(1 /\|y\|) y\left(k_{0}\right)\right|=1+$ $\left|x\left(k_{0}\right)\right|>1$ which is a contradiction. Thus no such $x$ and $y$ exist.

Assume $u \in \hat{c}_{0},\|u\|=1$ and $u$ is not an atom. Then for some $k_{0}$ in ${ }^{*} N$, $\left|u\left(k_{0}\right)\right|=1$ and $\theta=\max \left\{|u(k)|: k \neq k_{0}\right\}>0$. Let $x=u\left(k_{0}\right) \chi_{\left\{k_{0}\right\}}$ and $y=u-x$. Then $\|x\|=1,0<\|y\|=\theta \leqslant 1, x+y=u$, and $\|x+(1 / \theta) y\|=\|x-(1 / \theta) y\|$ $=1$.

Now assume that $\hat{c}_{0}$ is isometrically isomorphic to a hyperfinite-dimensional Banach space $\hat{W}$. Since every finite-dimensional space can be isometrically embedded in $l_{\infty}$ and by local reflexivity $l_{\infty}$ is finitely representable in $c_{0}$, we may assume that $W$ is a ${ }^{*}$-finite-dimensional subspace of ${ }^{*} c_{0}$. We endow $\hat{W}$ with the lattice ordering generated by the isometry, so we may consider atoms of $\hat{W}$, etc. In the following this is the ordering we consider on $\hat{W}$ and not the ordering $\hat{W}$ inherits as a subspace of $\hat{c}_{0}$.

Definition 1. For $n$ in ${ }^{*} N$ define $q \in W$ to be a $1 / n$-atom if

(i) $\|q\|=1$ and

(ii) for $1<m \leqslant n$ there do not exist $p_{1}, p_{2}$ in $W$ such that $\left\|p_{1}\right\|=1,1 / m<\left\|p_{2}\right\|$ $<1,\left\|p_{1}+p_{2}-q\right\|<1 /(3 m), 1-1 /(3 m)<\left\|p_{1}-\left(1 /\left\|p_{2}\right\|\right) p_{2}\right\|<1+1 /(3 m)$ and $1-1 /(3 m)<\left\|p_{1}+\left(1 /\left\|p_{2}\right\|\right) p_{2}\right\|<1+1 /(3 m)$.

Note that the definition of $1 / n$-atom is internal.

LEMMA 2. If $e$ is an atom of $\hat{W}$ of norm $1, n$ is a standard positive integer and $q \in W$ with $\|q\|=1$ and $\pi(q)=e$, then $q$ is $a 1 / n$-atom.

Proof of Lemma 2. We have assumed $\|q\|=1$. Suppose for some standard $m$ there exist $p_{1}$ and $p_{2}$ satisfying the conditions of (ii) of Definition 1 .

For some $k_{0}$ in ${ }^{*} N,\left|q\left(k_{0}\right)\right|=1$. Now since $\left\|\pi\left(p_{1}\right)\right\|=1$, there exists an atom $e^{\prime}$ in $\hat{W}$ of norm 1 such that $\left\|P_{e^{\prime}}\left(\pi\left(p_{1}\right)\right)\right\|=1$. (Here $P_{e^{\prime}}$ is the natural projection of $\hat{W}$ onto the one-dimensional subspace spanned by $e^{\prime}$. If $\Phi$ is the isometry of $\hat{c}_{0}$ onto $\hat{W}$ and $\Phi\left(\chi_{\{j\}}\right)=e^{\prime}$, then the corresponding projection in $\hat{c}_{0}$ sends $x$ to $x(j) \chi_{\{j\}}$.) Suppose $e \perp e^{\prime}$, i.e. $e$ and $e^{\prime}$ are the images under $\Phi$ of functions with distinct one-point supports. Let $P_{e^{\prime}}\left(\pi\left(p_{1}\right)\right)=s e^{\prime}$ and $P_{e^{\prime}}\left(\pi\left(p_{2}\right)\right)=t e^{\prime}$. Since $P_{e^{\prime}}(e)=$ $P_{e^{\prime}}(\pi(q))=0$, it follows that $\left\|s e^{\prime}+t e^{\prime}\right\|<1 /(3 m)$ and so $|s+t|<1 /(3 m)$. Since $|s|=1$, we have that $s$ and $t$ have opposite signs, $|t|>1-1 /(3 m)$ and $|s-t|>2$ $-1 /(3 m)>2-1 /(2 m)$. Hence, with st the standard part operation, we have

$$
\text { st }\left\|p_{1}-\left(1 /\left\|p_{2}\right\|\right) p_{2}\right\|>\left|s-\left(1 / \mathrm{st}|| p_{2} \|\right)\right|>|s-t|>2-1 /(2 m)>1+1 /(3 m)
$$

which is a contradiction.

So $\left\|P_{e}\left(\pi\left(p_{1}\right)\right)\right\|=1$ and $\left\|P_{e}\left(\pi\left(p_{2}\right)\right)\right\|<1 /(3 m)$. Since $\left\|p_{2}\right\|>1 / m$ it follows that $\left\|\pi\left(p_{2}\right)\right\|>1 / m$. Thus for some atom $e^{\prime \prime}$ of norm 1 which is orthogonal to $e$, $\left\|\pi\left(p_{2}\right)\right\|=\left\|P_{e^{\prime \prime}}\left(\pi\left(p_{2}\right)\right)\right\| \geqslant 1 / m$ and therefore $\left\|P_{e^{\prime \prime}}\left(\pi\left(p_{1}\right)\right)\right\|>2 /(3 m)$. Then for some choice of $\operatorname{sign} \beta$

$$
\left\|P_{e^{n}}\left(\pi\left(p_{1}\right)\right)+\left(\beta /\left\|\pi\left(p_{2}\right)\right\|\right) P_{e^{n}}\left(\pi\left(p_{2}\right)\right)\right\|>1+2 /(3 m) .
$$

It follows that $\left\|p_{1}+\left(\beta /\left\|\pi\left(p_{2}\right)\right\|\right) p_{2}\right\|>\alpha$ where $\alpha$ is standard with $1+1 /(3 m)<$ $\alpha<1+1 /(2 m)$. Since st $\left\|p_{2}\right\|>0$, we have 


$$
\beta /\left\|p_{2}\right\|={ }_{1} \beta /\left\|\pi\left(p_{2}\right)\right\| \text { and }\left\|p_{1}+\left(\beta /\left\|p_{2}\right\|\right) p_{2}\right\|>1+1 /(3 m)
$$

which is a contradiction.

LEMMA 3. If $q \in W$ is $a 1 / n$-atom for some infinite integer $n$, then $\pi(q)$ is $a$ norm-1 atom of $\hat{W}$.

Proof of Lemma 3. Assume to the contrary that $\pi(q)$ is not a norm-1 atom. Then by Lemma 1 there exist $x, y$ in $\hat{W}$ so that $x+y=\pi(q),\|x\|=1,0<\|y\|<$ $1,\|x+(1 /\|y\|) y\|=\|x-(1 /\|y\|) y\|=1$. Pick a standard $m$ such that $\|y\|>$ $1 / m$. Now pick $p_{1}, p_{2} \in W$ such that $\left\|p_{1}\right\|=1,\left\|p_{2}\right\|=\|y\|, \pi\left(p_{1}\right)=x$ and $\pi\left(p_{2}\right)=y$. Then $\left\|p_{1}+p_{2}-q\right\|<1 /(3 m), 1-1 /(3 m)<\left\|p_{1}+\left(1 /\left\|p_{2}\right\|\right) p_{2}\right\|<1$ $+1 /(3 m), 1-1 /(3 m)<\left\|p_{1}-\left(1 /\left\|p_{2}\right\|\right) p_{2}\right\|<1+1 /(3 m)$ which contradicts the definition of $1 / n$-atom since $\left\|p_{2}\right\|>1 / m$ and $m<n$.

Definition 2. If $n \in{ }^{*} N$ and $S$ is an internal set of $1 / n$-atoms, then $S$ is said to be acceptable if (i) $p \in S$ implies $-p \in S$ and (ii) $p_{1}, p_{2} \in S$ with $p_{1} \neq p_{2}$ implies $\left\|p_{1}-p_{2}\right\|>1 / 2$.

Note that if $p$ is a $1 / n$-atom then $-p$ is also a $1 / n$-atom. Further note that the definition of acceptable is internal. Since $W$ is a *-finite-dimensional space, there exists $r$ in ${ }^{*} N$ such that if $U$ is an internal subset of the unit ball of $W$ with the property that for every distinct pair $u_{1}, u_{2}$ in $U,\left\|u_{1}-u_{2}\right\|>1 / 2$, then the *-cardinality of $U$ is less than $r$. In particular every acceptable set of $1 / n$-atoms (for any $n$ ) has *-cardinality less than $r$.

Now let $n$ be a finite integer and let $S_{n}$ be an acceptable set of $1 / n$-atoms of maximal *-cardinality.

Lemma 4. If $q \in W$ with $\|q\|=1$, then there exists $p \in S_{n}$ such that $\|p+q\|>$ $5 / 4$.

Proof. Suppose, to the contrary, for some $q$ of norm 1 in $W,\|p+q\|<5 / 4$ for all $p$ in $S_{n}$. There exists a norm-1 atom $e$ in $\hat{W}$ such that $P_{e}(\pi(q))=e$. Pick $z$ in $W$ of norm 1 such that $\pi(z)=e$. Then $z$ is a $1 / n$-atom by Lemma 2. Suppose $\|z-p\|<1 / 2$ for some $p$ in $S_{n}$. Since there exists $k$ such that $|q(k)+z(k)|={ }_{1} 2$, we have $|q(k)|={ }_{1} 1,|z(k)|={ }_{1} 1$ and both have the same sign. Now $|z(k)-p(k)|$ $<1 / 2$, so $|q(k)+p(k)|>|q(k)+z(k)|-|z(k)-p(k)|>2-1 / 2-\varepsilon$ for any positive real $\varepsilon$. Hence $|q(k)+p(k)|>5 / 4$ and $\|q+p\|>5 / 4$ which is a contradiction. Since $p \in S_{n}$ implies $-p \in S_{n}$, we have $\| z-p \mid>1 / 2$ and $\|-z-p\|$ $>1 / 2$ for all $p \in S_{n}$, which implies $S_{n} \cup\{z,-z\}$ is an acceptable set of $1 / n$ atoms. This contradicts the maximality of $S_{n}$ and proves the lemma.

Now for every standard $n$ there is an acceptable set $S_{n}$ of $1 / n$-atoms such that if $q \in W$ and $\|q\|=1$, there exists $p \in S_{n}$ such that $\|q+p\|>5 / 4$. Since this describes an internal property of $n$, there exists $\omega \in{ }^{*} N \sim N$ and an acceptable set $S_{\omega}$ of $1 / \omega$-atoms such that for every $q$ in $W$ of norm 1 there exists $p$ in $S_{\omega}$ such that $\|q+p\|>5 / 4$. By Lemma 3 if $p \in S_{\omega}$ then $\pi(p)$ is a norm-1 atom of $\hat{W}$. Suppose $e$ is a norm-1 atom of $\hat{W}$ which is not of this form. Then $\|e+\pi(p)\|=1$ for all $p$ in $S_{\omega}$ which is impossible, since if $q \in W$ of norm 1 such that $\pi(q)=e$, then 
$\|q+p\| \geqslant 5 / 4$ for some $p$ in $S_{\omega}$. Thus $\left\{\pi(p): p \in S_{\omega}\right\}$ is exactly the set of norm-1 atoms in $\hat{W}$.

Let $T$ be an internal subset of $S_{\omega}$ such that for each $p$ in $S_{\omega}$ exactly one of $p$ and $-p$ is in $T$. Since $T$ is *-finite, there is a ${ }^{*}$-finite enumeration of $T$-say $p_{1}$, $p_{2}, \ldots, p_{\theta}$. For each $j, 1<j<\theta$, pick $k_{j}$ such that $\left|p_{j}\left(k_{j}\right)\right|=1$. Note that if $j_{1} \neq j_{2}$, then $p_{j_{1}}\left(k_{j_{2}}\right)={ }_{1} 0$. (Again we may assume that the map $j \mapsto k_{j}$ is internal; set $k_{j}$ to be the first $k$ such that $\left|p_{j}(k)\right|=1$.)

Suppose $\pi(q) \in \hat{W}$ and $\|\pi(q)\|=1$. Then for some $p_{j}$ in $S_{\omega}$ we have $\| \pi\left(p_{j}\right)+$ $\pi(q) \|=2$ and $\left\|\pi(q)-2 \pi\left(p_{j}\right)\right\|=1$. If $q\left(k_{j}\right) \neq 1 p\left(k_{j}\right)$, then $\left|\operatorname{st}\left(q\left(k_{j}\right)-2 p\left(k_{j}\right)\right)\right|>1$ which is a contradiction. Thus, in particular, if $\|q\|=1$, then $\left|q\left(k_{j}\right)\right|={ }_{1} 1$ for some $j$.

Define a ${ }^{*}$-linear map $I$ of $W$ into ${ }^{*}$-span $\left\{e_{k,}: 1<j<\theta\right\}$ by

$$
I(q)(k)=\left\{\begin{array}{l}
q(k) \text { if } k=k_{j} \text { for some } j, \\
0 \text { otherwise. }
\end{array}\right.
$$

By the remarks above $I$ is invertible and $\mathrm{st}\|I\|=\mathrm{st}\left\|I^{-1}\right\|=1$. If $W^{\prime}$ is the image of $W$ under $I$, then $\hat{W}$ is isometrically isomorphic to $\left(W^{\prime}\right)^{\wedge}$ under the map $\hat{I}$ defined by $\hat{I}(\pi(q))=\pi(I(q))$. Hence we may assume that for every $q$ in $W$ we have that the support of $q$ is contained in $\left\{k_{1}, k_{2}, \ldots, k_{\theta}\right\}$.

Now if $q \in W$ and $\|q\| \leqslant 1$ define $\sigma(q)=\left\{k_{j}:\left|q\left(k_{j}\right)\right|>3 / 4\right\}$. Let $l=$ the maximum *-cardinality of $\sigma(q)$ such that $\|q\|<1$ and $k_{j}$ not in $\sigma(q)$ implies $\left|q\left(k_{j}\right)\right|<1 / 4$. We show $l=\theta$. To see this let $w$ be an element of $W$ with $\|w\|<1$, *-cardinality of $\sigma(w)=l$ and $\left|w\left(k_{j}\right)\right| \leqslant 1 / 4$ for all $k_{j}$ not in $\sigma(w)$. Thus for every norm-1 atom $e$ in $\hat{W}$ either $\left\|P_{e}(\pi(w))\right\|>3 / 4$ or $\left\|P_{e}(\pi(w))\right\|<1 / 4$. Let $z$ be the preimage of $\pi(w)$ under the isometry between $\hat{W}$ and $\hat{c}_{0}$. Then $\|z\|<1$ and for each $k$ either $|z(k)|>3 / 4$ or $|z(k)|<1 / 4$. Thus $\left\{k \in{ }^{*} N:|z(k)|>3 / 4\right\}$ is *-finite, so there exists $z^{\prime}$ in $\hat{c}_{0}$ such that $\left|z^{\prime}(k)\right|=1$ if $|z(k)|>3 / 4$ and $z^{\prime}(k)=0$ otherwise. It follows that there exists $x$ in $\hat{W}$ of norm 1 such that $\left\|P_{\pi(p, j)}(x)\right\|=1$ if $k_{j} \in \sigma(w)$ and $\left\|P_{\pi\left(p_{j}\right)}(x)\right\|=0$ otherwise, i.e., if $\pi(u)=x$ with $\|u\|=1$, then $u\left(k_{j}\right)==_{1} 1$ for $k_{j}$ in $\sigma(w)$ and $u\left(k_{j}\right)={ }_{1} 0$ otherwise. Suppose $\sigma(w) \neq$ $\left\{k_{1}, k_{2}, \ldots, k_{\theta}\right\}$, say $m$ is such a $k_{j}$ and $u(m)={ }_{1} 0$. Let $v=(7 / 8) u+(7 / 8) p$, where $p \in T$ and $|p(m)|=1$. Then $|v(k)|={ }_{1} 7 / 8$ for $k$ in $\sigma(w) \cup\{m\}$ and $v(k)={ }_{1} 0$ otherwise. Since $\|v\|<1$ we have a contradiction to the maximality of $\sigma(w)$. Hence $\sigma(w)=\left\{k_{1}, k_{2}, \ldots, k_{\theta}\right\}$. But then we have $\pi(w)$ in $\hat{W}$ such that $\|\pi(w)\|<1$ and for every $x$ of norm 1 in $\hat{W}$, either $\|\pi(w)+x\|>7 / 4$ or $\|\pi(w)-x\|>7 / 4$, which is a contradiction. Thus there is no isometry of $\hat{c}_{0}$ onto a hyperfinite-dimensional Banach space.

COROLLARY. There is a positive bounded sentence $\sigma$ and an integer $n$ such that the approximating sentence $\sigma_{n}^{+}$is true in $c_{0}$ but false in every finite-dimensional Banach space.

The proof of this follows immediately from the Main Theorem and the results of [4] cited in the Introduction. Note that no explicit $\sigma$ has been obtained.

It remains an open question whether for various other spaces $E$ (e.g., $L^{1}[0,1]$ or 
$C[0,1])$, it is possible to have $\hat{E}$ isometrically isomorphic to a hyperfinite-dimensional Banach space. Perhaps a more interesting question is whether for every Banach space $E$ there is a nonstandard hull $\hat{E}$ which is isomorphic (not necessarily isometric) to a hyperfinite-dimensional Banach space. For $\hat{c}_{0}$ Henson shows in [3] that if $* \mathscr{N}$ has the $\kappa_{1}$-isomorphism property, then $\hat{c}_{0}$ is isomorphic to $\hat{l}_{\infty}(\omega)$ for any infinite integer $\omega$, where $\hat{l}_{\infty}(\omega)$ is $\hat{W}$ with $W$ the *-finite-dimensional subspace of ${ }^{*} l_{\infty}$ (and ${ }^{*} c_{0}$ ) of elements $p$ with support in $\{1,2, \ldots, \omega\}$. Indeed it follows from various results in [3] and [4] that every $L^{p}(\mu)$ space $(1<p<\infty)$ and every $C(K)$ space has a nonstandard hull which is isomorphic to a hyperfinite-dimensional Banach space.

\section{REFERENCES}

1. David Cozart and L. C. Moore, Jr., The nonstandard hull of a normed space, Duke Math. J. 41 (1974), 263-275.

2. Stefan Heinrich, Ultraproducts in Banach space theory, J. Reine Angew. Math. 313 (1980), 72-104.

3. C. Ward Henson, The isomorphism property in nonstandard analysis and its use in the theory of Banach spaces, J. Symbolic Logic 39 (1974), 717-731.

4. __ Nonstandard hulls of Banach spaces, Israel J. Math. 25 (1976), 108-144.

5. Keith Stroyan and W. A. J. Luxemburg, Introduction to the theory of infinitesimals, Academic Press, New York, 1976.

Departmgent of Mathematics, Duke Universtty, Durham, North Carolina 27706 